\title{
Probiotic Lactobacillus rhamnosus GG therapy and microbiological programming in infantile colic: a randomized, controlled trial
}

\author{
Anna Pärtty', Liisa Lehtonen'1, Marko Kalliomäki' ', Seppo Salminen² and Erika Isolauri'
}

BACKGROUND: Probiotic Lactobacillus reuteri and reduced allergen load may lessen the daily crying of colic infants, but the role of Lactobacillus rhamnosus GG (LGG) has remained obscure.

METHODS: Infants with colic $(n=30)$ were enrolled during the first 6 wk of life. All families received behavioral support and allergen avoidance diet: breastfeeding mothers followed cow's milk elimination diet and formula-fed infants received extensively hydrolyzed casein formula. The randomized, doubleblind intervention employed of LGG $4.5 \times 10^{9} \mathrm{cfu} / \mathrm{d}$ or placebo for a 4-wk study period. Daily crying was recorded by diaries and parental interviews. Fecal calprotectin and gut microbiota composition by quantitative PCR were evaluated before and after the intervention.

RESULTS: Daily crying time was comparable between the probiotic (173 min) and the placebo group (174 min; $P=0.99$ ) at the end of the intervention according to the parental diary. However, parents reported a decrease of 68\% (95\% confidence interval (Cl): 58-78) in daily crying in the probiotic and $49 \%$ (95\% Cl: 32-66) in the placebo group ( $P=0.05)$.

CONCLUSION: LGG in infants treated in tandem with behavioral support and a cow's milk elimination diet did not provide additional treatment effect for diary-verified colic crying although parental report of crying suggested the probiotic intervention effective.

O ne in five families encounter infant colic, defined as excessive and inconsolable crying exceeding $3 \mathrm{~h}$ a day on $3 \mathrm{~d}$ or more in $1 \mathrm{wk}(1,2)$. The colic period peaks at $6 \mathrm{wk}$, disappearing gradually around $12 \mathrm{wk}$ of age (3). Maternal emotional distress, anxiety, depression, and even child abuse may ensue, the entire family thus needing support during this stressful period (4-7). Such families should be recognized and ensured early referral for medical evaluation, as excessive crying is an unspecific symptom and might indicate some underlying medical problem (8). Further, with data accumulating to suggest that infant colic is not always benign and transient, but possible associated with subsequent problems such as functional gastrointestinal disorders, allergic disease, migraine, and psychological disorders (9-11), early recognition and counseling might also prove beneficial.

There are three main approaches to the problem: behavioral support for the family, including professional follow-up of the infant (12); a cow's milk elimination diet either for the breastfeeding mother or as an extensively hydrolyzed casein formula for the infant (13-15); and administration of probiotics $(16,17)$. To date, however, no consensus prevails on the lastmentioned strategy, and no study has combined these different approaches.

To test a comprehensive strategy, we evaluated a doubleblind placebo-controlled intervention trial of Lactobacillus rhamnosus GG (ATCC 53103, LGG) in the treatment of colic infants.

\section{RESULTS}

A total of 30 out of 34 evaluated infants met the inclusion criteria and were equally distributed between the intervention groups (Figure 1). All except one were Caucasian, mean (range) gestational age and birth weight $40 \mathrm{wk}(37$ to 42 ) and $3,400 \mathrm{~g}(2,370-4,425)$, respectively. Baseline characteristics are shown in Table 1. The 4-wk follow-up was completed by all. There were no study product-related adverse effects.

Before the intervention, the mean (range) daily crying time was $310 \mathrm{~min}$ (180 to 720 ) according to diary data. Crying time was comparable between the probiotic and placebo groups by both parental interviews and diary data (Table 2). All crying data are shown in Table 2 and Figure 2.

The mean (SD) weight gain during the study was comparable between the two groups, $867 \mathrm{~g}$ (326) vs. $941 \mathrm{~g}$ (308), respectively; $P=0.54$. Gastrointestinal function (regurgitation and stool frequency) were likewise comparable at enrolment. However, the number of daily regurgitations increased more often in the placebo than in the probiotic group during the follow-up ( 27 vs. $73 \%$ reported an increase, odds ratio (OR): 6.19; 95\% confidence interval (CI): 1.36-38.04; $P=0.04)$. Stool frequencies remained comparable (data not shown). Three (10\%) infants were admitted to hospital overnight due 


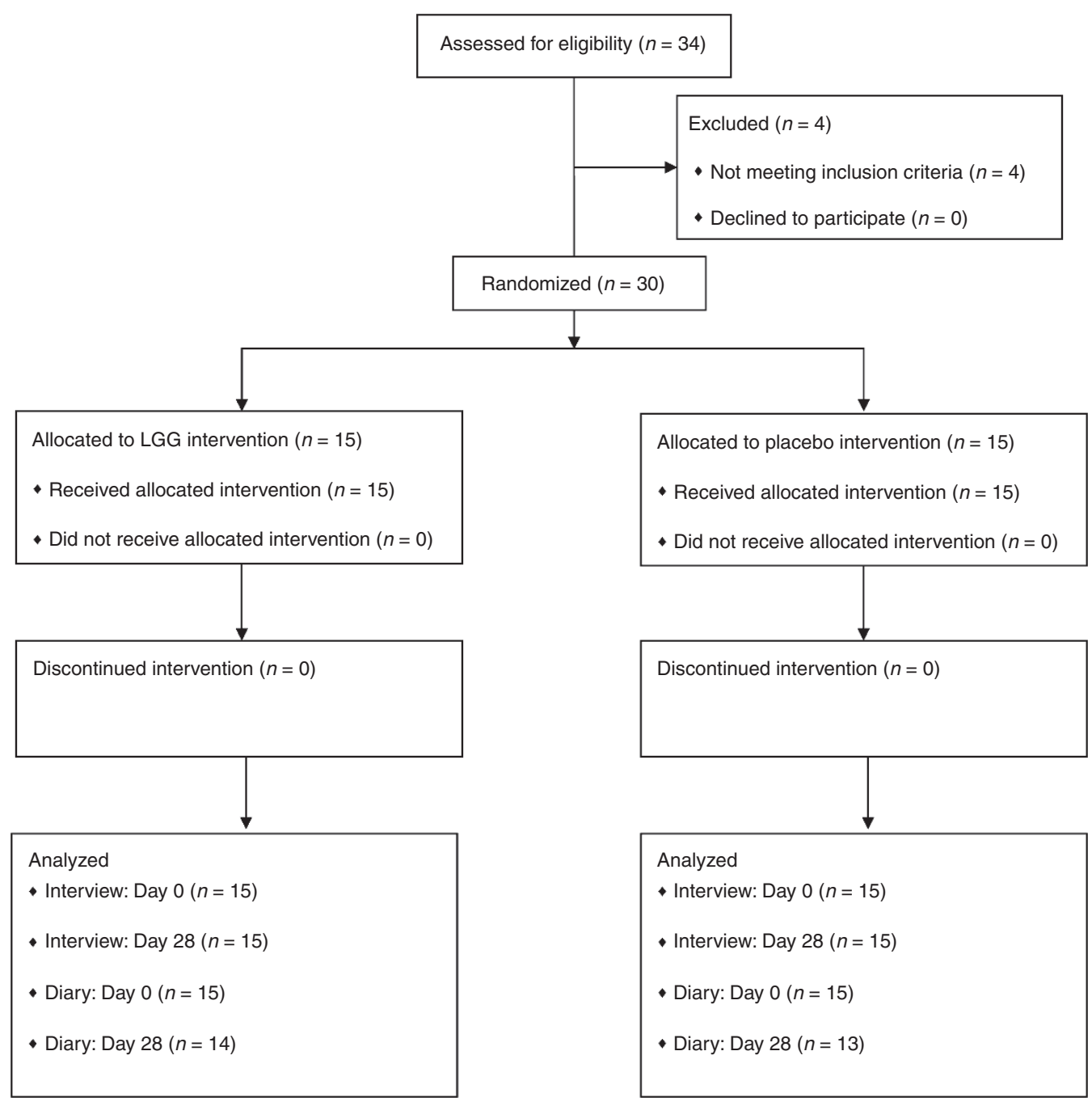

Figure 1. Flow chart of the study.

to parental exhaustion. All skin prick tests were negative. Challenge-proven cow's milk allergy was diagnosed after the study period in one $(6.7 \%)$ child in the LGG and in three (20.0\%) children in the placebo group (OR: 3.50; 95\% CI: $0.32-38.23 ; P=0.70)$. The intervention had no effect on fecal calprotectin levels (95\% CI for group difference: -270 to 270 ; $P$ $=1.00)$ (data not shown).

There was no statistical difference in gut microbiota composition between infants receiving LGG or placebo either at baseline or after intervention. However, an LGG effect was seen in an increase in different Bifidobacterium species. The number of B. lactis increased during the study in $47 \%$ of infants receiving LGG compared to $13 \%$ placebo (OR: 0.18 ; $95 \%$ CI: $0.04-0.77$; $P=0.02)$. Again, Bifidobacterium breve increased in $40 \%$ of infants receiving LGG compared with $13 \%$ of those receiving placebo (OR: 0.18 ; $95 \% \mathrm{CI}$ : $0.03-1.05 ; P=0.05$ ). A similar tendency was found in the number of $B$. adolescentis in stools (increase in $27 \%$ of probiotic recipients and in $7 \%$ in the placebo group; OR: 0.16 ; $95 \%$ CI: $0.02-1.59 ; P=0.10$ ). In general, Bifidobacterium brought out a distinction between colic infants and healthy controls, the median number of Bifidobacterium genus in stools being lower in the former $\left(1.1^{9}\right.$ vs. $4.6^{10}$ respectively; $P=0.04$ ). Moreover, colic infants tended to be less frequently colonized with $B$. breve than healthy infants at baseline (13 vs. $50 \%$, respectively; OR: 3.77 ; $95 \%$ CI: $1.04-13.65$; $P=$ 0.07 ), and at close of study despite the intervention (27 vs. $64 \%$, respectively; OR: 1.52 ; 95\% CI: $0.67-3.44 ; P=0.06$ ).

\section{DISCUSSION}

Based on parental diaries on the duration of crying, colic infants do not benefit from LGG in tandem with behavioral counseling and cow's milk elimination. However, parents reported in their interviews that daily crying decreased more in infants receiving LGG compared to placebo and, the number of cry days per week was lower and number of responders higher in the LGG group in comparison to placebo.

Two previous randomized double-blind placebo-controlled studies using Lactobacillus reuteri (DSM 17938) have shown a therapeutic effect $(16,17)$, while a mixture of different probiotics (L. rhamnosus GG, L. rhamnosus LC705, B. breve Bbi99, Propionibacterium freudenreichii ssp, and Shermanii JS or L. rhamnosus and Bifidobacterium infantis) has not proved 
successful $(18,19)$. Of note, our design differed from earlier setups in several respects, in addition to the difference in probiotic strain. First, we enrolled only infants younger than $6 \mathrm{wk}$ to coincide the maximum crying time in colic, while previous investigators enrolled infants up to 16 or $20 \mathrm{wk}$ of age $(16,17)$, a time span when crying might be of different etiology (12). Secondly, parents were referred to the study or independently sought participation if they perceived their infant's crying as colic. In previous studies, the colic diagnosis was based solely on daily crying amount $(16,17)$. Thirdly, in addition to cow's milk elimination, behavioral counseling and lactation support were offered to all. This is thus the first study in which all potentially effective strategies against colic (13-16) were

Table 1. Baseline characteristics of the participants

\begin{tabular}{lcc}
\hline & LGG $(n=15)$ & Placebo $(n=15)$ \\
\hline Mother & & \\
University education & $1(53)$ & $7(47)$ \\
Depression & $3(20)$ & $1(7)$ \\
Smoking & $3(20)$ & $0(0)$ \\
Probiotic use during pregnancy & & $7(47)$ \\
Infant & $4(27)$ & \\
Siblings & $12(80)$ & $12(80)$ \\
Family history of atopy & $9(60)$ & $10(67)$ \\
Perinatal antibiotic exposure & $13(87)$ & $12(80)$ \\
Vaginal delivery & $7(47)$ & $5(33)$ \\
Male & $3490(430)$ & $3320(520)$ \\
Birth weight (g) & $51.2(2.0)$ & $50.3(2.2)$ \\
Birth length (cm) & $38.0(10.8)$ & $34.8(9.9)$ \\
Age at entry (days) & $6(40)$ & $5(33)$ \\
Exclusive breastfeeding & $7(47)$ & $8(53)$ \\
Partly breastfeeding & $2(13)$ & $2(13)$ \\
Exclusively formula feeding & $11(73)$ & $8(53)$ \\
Earlier exposure to probiotics & $4(27)$ & $3(20)$ \\
LGG & $7(47)$ & $5(33)$ \\
Lactobacillus reuteri & & \\
\hline
\end{tabular}

Results are given as mean (SD) or as number (\%) of subjects. All the characteristics were comparable between the study groups $(P>0.05)$. Comparisons were made using chisquare test, Fisher's exact test, or independent samples $t$-test as appropriate.

LGG, Lactobacillus rhamnosus GG. equally in use in order to assess a specific benefit, if any, from LGG intervention.

Two different means of evaluating the effect of probiotics in infant crying were applied: a validated, prospective detailed cry diary and an interview on parental recall regarding crying during the previous week. The diary was assumed to be objective measuring the quantity of crying while parental recall may include some qualitative features of crying not captured by a diary affecting subjective experience and thus reflecting the real relevance of crying to the parents. The inclusion of this subjective perspective presumably adds a wider dimension to the potential effect of the intervention. Interestingly, the effect was not evident in diary data but only in parental interview. The discrepancy remains unexplained. In any case, parents' subjective perception of infant crying and of a potential effect of any colic intervention is clinically significant since it is parents' perception of colic which leads to referrals.

We regard good compliance as another strength of the study: all families attended the follow-up and returned stool samples as scheduled. In accord with earlier studies, we found the number of total bifidobacteria in stool samples to be lower in colic infants compared to the reference population $(20,21)$. We chose as reference a population presumably representing a model of infant feeding and health: a vaginally delivered breastfed child free of signs and symptoms of disease in follow-up. In contrast to our earlier cohort, $B$. breve was not associated with excessive crying, this underlining the complexity of infant gut microbiota composition during its maturation process and the intricacy of any concept of normal colonization (21).

Previous probiotic intervention studies in colic infants report increased lactobacilli and bifidobacteria, but a decrease in Escherichia coli $(16,18)$. However, only one study could associate these alterations with a reduction in crying (16). The present intervention promoted an increase in B. lactis numbers. One possible mechanism in the impact of probiotics on infant colic is regulation of gut inflammation possibly induced by aberrant microbiota or sensitization to specific food antigens. Probiotics may degrade antigens to more tolerogenic form and thus balance the generation of pro- and anti-inflammatory cytokines (22). Preliminary data suggest that specific probiotics may promote oral tolerance induction (23). This might open up new insights into the long-term sequelae of infant colic as well as other gut-associated disorders in which a microbial etiology is beginning to emerge.

Table 2. The crying characteristics of the infants in the placebo and probiotic groups

\begin{tabular}{|c|c|c|c|c|c|}
\hline & & \multicolumn{2}{|c|}{ Mean daily crying time (SD) } & \multirow{2}{*}{$\begin{array}{c}\text { Mean daily crying time } \\
\text { decrease } \%(95 \% \mathrm{Cl})\end{array}$} & \multirow{2}{*}{$\begin{array}{c}\text { Number of } \\
\text { responders (\%) }\end{array}$} \\
\hline & & Day 0 & Day 28 & & \\
\hline Placebo & Interview $(n=15)$ & $262(82)$ & $138(108)^{*}$ & $49(32-66)^{* *}$ & $7(47)^{\dagger}$ \\
\hline \multirow[t]{2}{*}{ LGG } & Interview $(n=15)$ & $236(112)$ & $70(36)^{*}$ & $68(58-78)^{* *}$ & $13(87)^{\dagger}$ \\
\hline & Diary ${ }^{b}$ & $295(116)$ & $173(64)$ & $17(2-33)$ & $0(0)^{\ddagger}$ \\
\hline
\end{tabular}

${ }^{a} n=15$ on day $0 ; n=13$ on day $28 .{ }^{b} n=15$ on day $0 ; n=14 n$ day $28 .{ }^{*} P=0.03 ; * * P=0.0495 ;{ }^{\dagger} P=0.02 ;{ }^{\ddagger} P=0.04$. All the other characteristics were comparable between the study groups $(P>0.05)$. Comparisons were made using chi-square test, Fisher's exact test or independent samples $t$-test as appropriate.

$\mathrm{Cl}$, confidence interval; LGG, Lactobacillus rhamnosus GG. 


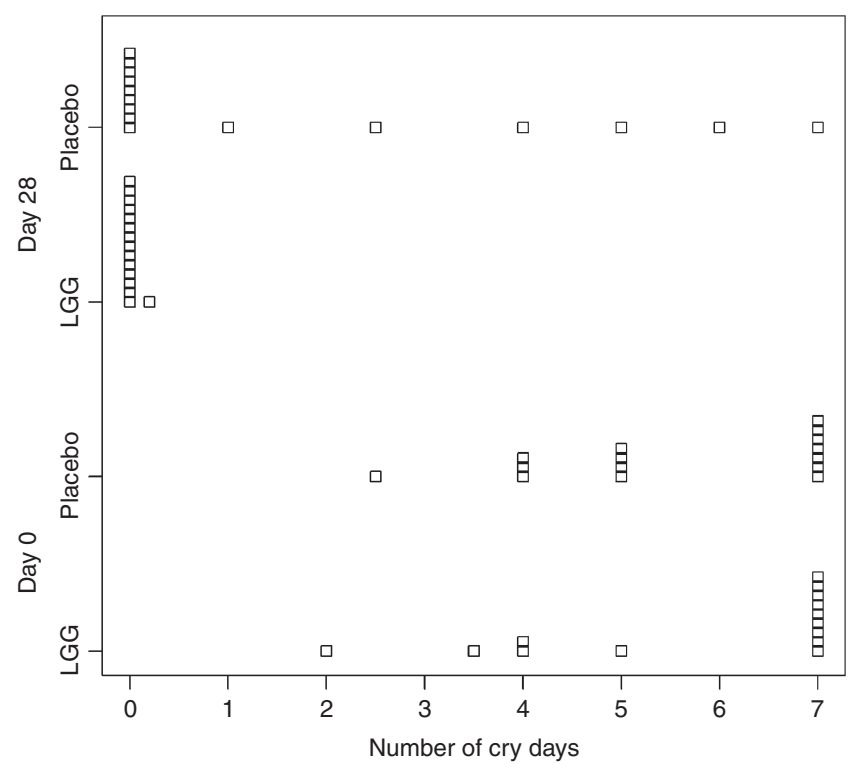

Figure 2. The number of days per week the infant cried for more than $3 \mathrm{~h} / \mathrm{d}$ in the LGG $(n=15)$ and placebo groups $(n=15)$ as reported by the parental interview on day 0 and day 28 . At day 28 the number of cry days was significantly higher in placebo group compared to LGG group $(p=0.02)$. Every box represents an infant. Comparisons were made using independent samples $t$-test. LGG, Lactobacillus rhamnosus GG.

In conclusion, we demonstrated that LGG supplementation in tandem with behavioral and nutritional counsel did not provide additional treatment effect for diary-verified colic crying.

\section{METHODS}

\section{Study Objectives and Outcomes}

Difference in daily average crying time between the LGG and placebo groups was selected as the primary outcome of the study. Daily crying time, number of responders (i.e., the infants whose crying time decreased at least the half), number of days the infant cried for more than $3 \mathrm{~h}$ (defined as cry days), and change in fecal calprotectin level were all selected as secondary outcomes. Comparison of gut microbiota both between the intervention groups and between colic infants and healthy reference population were other secondary outcomes. Mean weight gain, number of daily regurgitations, stool frequency, and allergic sensitization were also evaluated.

\section{Study Population}

Colic infants participating in this randomized, double-blind, placebo-controlled study were recruited in South-West Finland between February 2011 and January 2013. Advance information was given to families in maternity wards, well-baby clinics, and pediatric outpatient clinics in Turku University Hospital. Families interested contacted a research nurse. For enrolment, infants had to be full-term, under 6 wk of age exhibiting paroxysmal, unsoothable crying raising concern in the parents, crying at least $3 \mathrm{~h}$ per day on $3 \mathrm{~d}$ per week. Before the first study visit, parents were instructed to complete a diary for $3 \mathrm{~d}$ to assess the infant for eligibility (24). The infant was eligible if the diary recorded crying lasting least $180 \mathrm{~min}$ per day during the last $3 \mathrm{~d}$. Infants with diagnosed chronic diseases were excluded. The reference population comprised 11 matched controls from an on-going clinical trial with accurate prospective monitoring of potential and relevant confounding factors concerning gut microbiota (feeding type, mode of delivery, and use of probiotics and antibiotics) and recording of the daily crying $(25,26)$. The infants of the reference population were healthy, breastfed infants born in the same hospital by vaginal delivery at term and not exposed to antibiotics or probiotics during the neonatal period, cried less than $180 \mathrm{~min}$ per day, and provided fecal samples at $3(n=6)$ and/or $12 \mathrm{wk}(n=11)$ of age $(25,26)$.

\section{Study Design}

All families received counseling comprising general information on infant colic, supportive discussions with study personnel, and free access to nurse and physician, together with the possibility of an overnight hospital stay in the case of parental exhaustion. During the study visit, breastfeeding and bottle feeding techniques were evaluated by a certified consultant (Ulla-Maija Eriksson), and proper feeding techniques inculcated on request.

At entry, skin prick tests were performed to study IgE-mediated sensitization to common food allergens (cow's milk, raw hen's egg white, and wheat) as previously described (27). To assess the impact of food allergy on colic as defined in Finnish guidelines, all breastfeeding mothers were asked to avoid products containing cow's milk and egg, and formula-fed infants received extensively hydrolyzed casein formula (Nutramigen; Mead-Johnson, Evansville, IN) during the study period. Dietary adherence was confirmed by interview during follow-up visits. At close of the study, breastfeeding mothers resumed their normal diet and standard formula was re-introduced for formula-fed infants. In the case of suspected cow's milk allergy, a formal challenge was implemented at the Department of Pediatrics, Turku University Hospital.

The infants received orally probiotic LGG (Mead-Johnson, Evansville, IN) $4.5 \times 10^{9} \mathrm{cfu} / \mathrm{d}$ or placebo (microcrystalline cellulose; Tamro, Vantaa, Finland) for 28 d $(26,28,29)$. Breastfed infants received the product from closed sachets identical in appearance, taste, and smell. Parents were taught to open the sachets and mix the products immediately before administration in $\sim 10 \mathrm{ml}$ of breast milk given by spoon or bottle once a day. Formula-fed infants received LGG or placebo mixed with extensively hydrolyzed casein formula (NutramigenLGG or Nutramigen; Mead-Johnson). Nutramigen-LGG contains LGG $5.4 \times 10^{7} \mathrm{cfu} / \mathrm{g}$ of the product. In both cases, breastfed and formula-fed infants received a daily dose of $4.5 \times 10^{9} \mathrm{cfu}$ of LGG, achieved by an appropriate daily amount of sachets, formula, or combination.

Parents were instructed to inspect the product for signs of degradation before use and keep the sachets refrigerated and formula at room temperature. The LGG and placebo sachets were prepared by the study nurses in the University laboratory, and the viability of the probiotic was confirmed by regular blinded analysis in the Functional Foods Forum, University of Turku.

\section{Randomization}

Participants were randomly assigned by an independent statistician (J.M.) to one or other study group using the random allocation rule. Study personnel, hospital staff, and parents were blinded to the randomization. To ensure allocation concealment, a study group member not involved in the conduct or reporting of the study was responsible for packaging and labeling the products. The study nurse allocated the next available product on entry into the trial, and each patient received the product directly from the study clinic. The code was revealed to the investigator once recruitment, data collection, and analysis were completed. Data were collected on printed case record forms, and group members entered the data. All data were kept confidential.

\section{Assessment of Infant Crying}

To confirm eligibility, parents recorded their infants' behavior (sleeping, awake and content, fussing, crying, feeding) on three consecutive days prior to the intervention, using the validated Baby Day Diary (24). The 24-h day was divided into 5-min sections, and parents filled in the diary as convenient, usually during feeding. Instructions on record-keeping were given by the same research nurse (Ulla-Maija Eriksson). Recording was not completely successful in five cases by close of intervention: the diary not being returned in three cases and in two only $1 \mathrm{~d}$ being covered. Daily crying was estimated by parental interviews at entry and close of intervention. Crying was defined as "continuous crying" and fussing as "noncontinuous negative vocalization." Total distress included both. In interview, parents reported the infant's behavioral patterns, including sleeping patterns, crying (daily crying time in minutes, number of days per week on which crying extends more than $3 \mathrm{~h}$ ), feeding, regurgitation (at enrolment: number of regurgitations per day; on days 7 and 28: an increase, no change, a decrease compared to study entry), vomits, 
stools (consistency normal, loose, firm or hard, and frequency as 1/wk, 2-4/wk, 1-2/d, 3-4/d, >4/d), infections and other diseases, use of medications and parental coping. Specific items were administration and potential adverse effects of the study product. Family history of allergic and other diseases, parents' smoking, mother's education, mother's medication and use of probiotics during pregnancy and lactation, mother's diet, infant's gestational age, mode of delivery, birth characteristics, and earlier exposure to probiotics and medication were asked in interview at enrolment.

After the enrolment day (day 0), follow-up consultations were conducted by the same study nurse and physician on days 7 and 28, and when necessary during the study. A medical examination was made, and weight, length, and head circumference measured and growth charts drawn at each visit.

\section{Fecal Sample Collection and Analysis}

Fecal samples were collected from diapers on day 0 and 28, immediately frozen to $-20^{\circ} \mathrm{C}$ and delivered within $24 \mathrm{~h}$ to the study clinic, where they were stored at $-70{ }^{\circ} \mathrm{C}$ until analyzed.

\section{Calprotectin Assay}

Calprotectin was analyzed using the CALPROLAB Calprotectin ELISA (ALP) kit (CALP0170, Calpro AS, Lysaker, Norway) according to manufacturer's instructions.

Sample preparation used the weighting method as instructed. Supernatants from the fecal sample extraction procedures were diluted 1:100 and analyzed using enzyme-linked immunosorbent assay.

\section{DNA Isolation and Quantitative PCR Assay}

Samples were pretreated and DNA extracted using the InviMag Stool DNA kit (Stratec Molecular, Berlin, Germany) and the automated KingFisher DNA extraction system (Thermo Fisher Scientific Oy, Vantaa, Finland), as previously described (30). DNA samples were stored at $-20^{\circ} \mathrm{C}$ until analyzed. Quantitative PCRs were conducted as described elsewhere $(31,32)$. For quantitative PCR, the standard DNA was prepared as previously (30). Detection and PCR amplification were performed with an ABI PRISM 7300-PCR sequence detection system (Applied Biosystems, Foster City, CA).

\section{Statistics}

On the basis of a recent colic study with a probiotic intervention (16), sample size was calculated to find a difference of $55 \mathrm{~min}$ in daily average crying time between the groups. With $5 \%$ probability of type I error and $10 \%$ probability of type II error, and an estimated withingroup SD of $45 \mathrm{~min}, 15$ patients per group were needed.

Changes in the amount of microbiota between the two measurement points were categorized as any increase, no change, and any decrease, and the two groups compared using chi-square test for trend. Comparisons between two nominal variables were by chisquare or Fisher's exact test as appropriate. Microbiota values were compared using Mann-Whitney $U$-test, other continuous variables using independent samples $t$-test. The gut microbiota of the colic infants at enrolment and end of intervention was compared with controls at the age of 3 and $12 \mathrm{wk}$, respectively. Statistical analyses were made using SAS for Windows, version 9.2. $P$ values below 0.05 were considered statistically significant.

\section{Ethics Statement}

The study was approved by the Ethics Committee of the Hospital District of South-West Finland. Written informed consent was obtained from the parents of the infants. The trial was registered at ClinicalTrials.gov (http://www.clinicaltrials.gov/ct/gui/show/ NCT00167700).

\section{ACKNOWLEDGMENTS}

We would like to thank the families participating in our study; Jaakko Matomäki for statistical consultation, Robert MacGilleon for language review of the manuscript, and Ulla-Maija Erikson for help with the follow-up of participants.

\section{STATEMENT OF FINANCIAL SUPPORT}

The study was supported by the Foundation for Pediatric Research (Finland), an EVO grant from Turku University Hospital (Finland) and the Yrjö Jahnsson Foundation (Finland). The first author (A.P.) has received funding from the National Graduate School of Clinical Investigation (Finland).

Disclosure: The Mead Johnson Nutrition Company USA covered the costs of the probiotic products, provided the hydrolyzed formulas and part of the salary for the first author (Anna Pärtty). The Mead Johnson Company had no influence on the design or conduct of the study, data management and analysis, writing of the report, or the decision to submit the manuscript for publication.

\section{REFERENCES}

1. Alvarez M, St James-Roberts I. Infant fussing and crying patterns in the first year in an urban community in Denmark. Acta Paediatr 1996;85: 463-6.

2. Wessel MA, Cobb JC, Jackson EB, Harris GS Jr, Detwiler AC. Paroxysmal fussing in infancy, sometimes called colic. Pediatrics 1954;14:421-35.

3. Brazelton TB. Crying in infancy. Pediatrics 1962;29:579-88.

4. Räihä H, Lehtonen L, Korhonen T, Korvenranta H. Family life 1 year after infantile colic. Arch Pediatr Adolesc Med 1996;150:1032-6.

5. Miller AR, Barr RG, Eaton WO. Crying and motor behavior of six-weekold infants and postpartum maternal mood. Pediatrics 1993;92:551-8.

6. Akman I, Kusçu K, Ozdemir N, et al. Mothers' postpartum psychological adjustment and infantile colic. Arch Dis Child 2006;91:417-9.

7. Lee C, Barr RG, Catherine N, Wicks A. Age-related incidence of publicly reported shaken baby syndrome cases: is crying a trigger for shaking? J Dev Behav Pediatr 2007;28:288-93.

8. Freedman SB, Al-Harthy N, Thull-Freedman J. The crying infant: diagnostic testing and frequency of serious underlying disease. Pediatrics 2009;123:841-8.

9. Savino F, Castagno E, Bretto R, Brondello C, Palumeri E, Oggero R. A prospective 10-year study on children who had severe infantile colic. Acta Paediatr Suppl 2005;94:129-32.

10. Romanello S, Spiri D, Marcuzzi E, et al. Association between childhood migraine and history of infantile colic. JAMA 2013;309:1607-12.

11. Hemmi MH, Wolke D, Schneider S. Associations between problems with crying, sleeping and/or feeding in infancy and long-term behavioural outcomes in childhood: a meta-analysis. Arch Dis Child 2011;96:622-9.

12. James-Roberts IS. The Origins, Prevention and Treatment of Infant Crying and Sleeping Problems. An Evidence-Based Guide for Healthcare Professionals and the Families They Support. East Sussex, England: Routledge, 2012.

13. Arikan D, Alp H, Gözüm S, Orbak Z, Cifçi EK. Effectiveness of massage, sucrose solution, herbal tea or hydrolysed formula in the treatment of infantile colic. J Clin Nurs 2008;17:1754-61.

14. Lucassen PL, Assendelft WJ, Gubbels JW, van Eijk JT, Douwes AC. Infantile colic: crying time reduction with a whey hydrolysate: a double-blind, randomized, placebo-controlled trial. Pediatrics 2000;106:1349-54.

15. Hill DJ, Roy N, Heine RG, et al. Effect of a low-allergen maternal diet on colic among breastfed infants: a randomized, controlled trial. Pediatrics 2005;116:e709-15.

16. Savino F, Cordisco L, Tarasco V, et al. Lactobacillus reuteri DSM 17938 in infantile colic: a randomized, double-blind, placebo-controlled trial. Pediatrics 2010;126:e526-33.

17. Szajewska H, Gyrczuk E, Horvath A. Lactobacillus reuteri DSM 17938 for the management of infantile colic in breastfed infants: a randomized, double-blind, placebo-controlled trial. J Pediatr 2013;162:257-62.

18. Mentula S, Tuure T, Koskenala R, Korpela R, Könönen E. Microbial composition and fecal fermentation end products from colicky infants - a probiotic supplementation pilot. Microb Ecol Health D 2008;20:37.

19. Dupont C, Rivero M, Grillon C, Belaroussi N, Kalindjian A, Marin V. Alpha-lactalbumin-enriched and probiotic-supplemented infant formula in infants with colic: growth and gastrointestinal tolerance. Eur J Clin Nutr 2010;64:765-7.

20. de Weerth C, Fuentes S, Puylaert P, de Vos WM. Intestinal microbiota of infants with colic: development and specific signatures. Pediatrics 2013;131:e550-8. 
21. Pärtty A, Kalliomäki M, Endo A, Salminen S, Isolauri E. Compositional development of Bifidobacterium and Lactobacillus microbiota is linked with crying and fussing in early infancy. PLoS One 2012;7:e32495.

22. Schultz M, Linde HJ, Lehn N, et al. Immunomodulatory consequences of oral administration of Lactobacillus rhamnosus strain GG in healthy volunteers. J Dairy Res 2003;70:165-73.

23. Berni Canani R, Nocerino R, Terrin G, et al. Formula selection for management of children with cow's milk allergy influences the rate of acquisition of tolerance: a prospective multicenter study. J Pediatr 2013;163:771-7.e1.

24. Barr RG, Kramer MS, Boisjoly C, McVey-White L, Pless IB. Parental diary of infant cry and fuss behaviour. Arch Dis Child 1988;63:380-7.

25. Kalliomäki M, Laippala $\mathrm{P}$, Korvenranta H, Kero P, Isolauri E. Extent of fussing and colic type crying preceding atopic disease. Arch Dis Child 2001;84:349-50.

26. Kalliomäki M, Salminen S, Arvilommi H, Kero P, Koskinen P, Isolauri E. Probiotics in primary prevention of atopic disease: a randomised placebocontrolled trial. Lancet 2001;357:1076-9.
27. Nermes M, Niinivirta K, Nylund L, et al. Perinatal pet exposure, faecal microbiota, and wheezy bronchitis: is there a connection? ISRN Allergy 2013;2013:827934.

28. Hojsak I, Abdović S, Szajewska H, Milosević M, Krznarić Z, Kolacek S. Lactobacillus GG in the prevention of nosocomial gastrointestinal and respiratory tract infections. Pediatrics 2010;125:e1171-7.

29. Majamaa $\mathrm{H}$, Isolauri E. Probiotics: a novel approach in the management of food allergy. J Allergy Clin Immunol 1997;99:179-85.

30. Nylund L, Heilig HG, Salminen S, de Vos WM, Satokari R. Semi-automated extraction of microbial DNA from feces for $\mathrm{qPCR}$ and phylogenetic microarray analysis. J Microbiol Methods 2010;83:231-5.

31. Collado MC, Isolauri E, Laitinen K, Salminen S. Distinct composition of gut microbiota during pregnancy in overweight and normal-weight women. Am J Clin Nutr 2008;88:894-9.

32. Scalabrin DM, Mitmesser SH, Welling GW, et al. New prebiotic blend of polydextrose and galacto-oligosaccharides has a bifidogenic effect in young infants. J Pediatr Gastroenterol Nutr 2012;54:343-52. 